Original article

\title{
Antibacterial and antibiofilm effects of silver and gold nanoparticles on uropathogenic Escherichia coli
}

\author{
Rini Purbowati ${ }^{1 *}$, Sugiharto $^{2}$, Agusniar Furkani Listyawati ${ }^{3}$, Masfufatun $^{4}$, Lusiani Tjandra ${ }^{5}$, Noer Kumala Indahsari ${ }^{4}$ \\ ${ }^{1}$ Biomedical Department and Biomolecular Research, Faculty of Medicine, Wijaya Kusuma Surabaya University, Indonesia \\ ${ }^{2}$ Department of Public Health, Medical Faculty, University of Wijaya Kusuma Surabaya, Indonesia \\ ${ }^{3}$ Department of Microbiology and Parasitology, Medical Faculty, University of Wijaya Kusuma Surabaya, Indonesia \\ ${ }^{4}$ Department of Biochemistry, Medical Faculty of Medicine, University of Wijaya Kusuma Surabaya, Indonesia \\ ${ }^{5}$ Department of Pharmacology, Medical Faculty, University of Wijaya Kusuma Surabaya, Surabaya, Indonesia
}

\begin{abstract}
Nosocomial infections of the urinary tract are the most common nosocomial infections. Infectious diseases currently pose a life threatening due to the increasing phenomenon of infectious agent resistance. One of the factors known to cause resistance to broad-spectrum antimicrobials agent is the susceptibility to biofilm formation. The use of silver nanoparticles (NPs) is now regarded as one of the most promising strategies to target infections associated with biofilms. This study aims to determine the application of nanotechnology in the form of metal nanoparticles in targeting the formation of biofilms on infectious agents. The antibacterial and antibiofilm activity test was performed on a 96-well microtiter plate on uropathogenic Escherichia coli (UPEC) at concentrations of $0,10,20,30,40,50,60,70,80,90$, and $100 \mathrm{ppm}$. The results showed the addition of silver and gold nanoparticles at different concentrations affected and inhibited the growth of UPEC and prevented the formation of biofilms, as indicated by a decrease in the OD value. In conclusion, silver and gold nanoparticles have antibacterial and antibiofilm effects on UPEC.
\end{abstract}

Abstract

Keywords: Gold, silver, nanoparticle, biofilm, UPEC

Received: September 9, 2021 Revised: December 24, 2021 Accepted: December 25, 2021

\section{Introduction}

Infectious diseases are currently a major concern due to the high morbidity and mortality rates posed by infectious agents (Hashimoto et al., 2017), one of these is Urinary Tract Infection (UTI). Nosocomial infections of the urinary tract are the most frequent nosocomial infections, accounting for $30-40 \%$ of the incidence (Kamat et al., 2009). Based on reported data, about 50$60 \%$ of adult women have experienced a UTI in their lifetime (Medina \& Castillo-Pino, 2019)

Infectious diseases currently pose a threat to life due to the phenomenon of resistance of infectious agents to nearly all drugs used. One of the factors known to cause resistance to broad-spectrum antimicrobials is the ability to form biofilms (Hashimoto et al., 2017). A biofilm is a multicellular consortium of microbial cells adhered to a surface and enveloped by a self-secreting extracellular polymer matrix. Bacteria in biofilms are usually more resistant to antimicrobial agents (Zhang, 2017). The emergence of the phenomenon of resistance of infectious agents to antimicrobials requires the search for new therapeutic agents that are effective in combating drugresistant pathogens (Hashimoto et al., 2017).

Several strategies are known to control and overcome biofilms from pathogens, including antimicrobial peptides, biofilm-degrading enzymes, quorum sensing

* Corresponding Author:

Rini Purbowati

Biomedical Department and Biomolecular Research, Faculty of Medicine Wijaya Kusuma Surabaya University, Indonesia Phone: +6281333113273

E-mail: purbowatirini@gmail.com; rini.purbowati@uwks.ac.id
(QS) inhibitors, essential oils, and nanotechnology/nanoparticles (NPs). Nanotechnology offers opportunities for infection control. These nanomaterials have unique properties compared to their natural counterpart, such as their large volume-to-surface area ratio and high flexibility, which can be exploited. In the last decade, billions of dollars have been invested in the development of nanomaterials for medicine in the global market (Vallet-Regí et al., 2019).

Nanoparticles are materials with a diameter of 1 to $100 \mathrm{~nm}$ that can be made from a variety of materials, such as copper, zinc, titanium, magnesium, gold, alginate, and silver. The use of silver nanoparticles (NPs) is now regarded as one of the most promising strategies to combat infection caused by biofilms associated with medical devices embedded in patients' bodies (Oliveira et al., 2011). It has been reported that (NPs) (e.g., silver/silver, zinc oxide/zinc oxide, copper oxide/copper oxide) exhibit antibacterial properties. Gold nanoparticles (AuNPs) exhibit antimicrobial effects through several mechanisms, including adhesion to the surface of bacterial cell walls and membranes, penetration into cells and disruption of intracellular organelles and biomolecules, induction of oxidative stress, and modulation of signal transduction pathways. Silver nanoparticles (AgNPs) may also act as carriers to transport $\mathrm{Ag}^{+}$more efficiently to bacterial cells, whose proton-motive force consequently lowers local $\mathrm{pH}$ and increases $\mathrm{Ag}^{+}$release. In addition, silver nanoparticles are thought to form free radicals upon contact with bacteria and damage cell membranes by making them porous. However, other researchers suggest that AgNPs attach to the bacterial surface and alter the properties of 
the membrane, while they may cause DNA damage within bacterial cells (Anees Ahmad et al., 2020)

In Indonesia, research on the use of nanoparticles as antibiofilm agents is still few and limited to certain microbes and the analysis is still simple. Therefore, an in-depth study of the potential of nanoparticles as antibiofilm agents in infectious agents derived from clinical isolates is needed. Although AgNPs are the most commonly used metal nanoparticles in the medical field to treat infections, the understanding of the exact mechanism of action on microbes is still unclear (ValletRegí et al., 2019). Based on above described context, this study was aimed to scientifically investigate the effect of nanotechnology application in the form of metal nanoparticles in influencing the formation of biofilms on infectious agents.

\section{Methods}

\section{Preparation of UPEC inoculum}

The UPEC used in this study in the study was obtained from the previous isolation (Kitagawa et al., 2018). The UPEC was grown on nutrient agar (NA) medium for $24 \mathrm{~h}$ incubation at $37^{\circ} \mathrm{C}$. The UPEC subculture was grown on Luria Bertani (LB) medium for $24 \mathrm{~h}$ incubation at $37^{\circ} \mathrm{C}$. Subsequently, the culture was centrifuged at $5000 \mathrm{rpm}$ for $5 \mathrm{~min}$. The supernatant was resuspended with $0.9 \% \mathrm{NaCl}$ and adjusted to $\mathrm{OD}_{490}=0.5$, then used as an inoculum in each treatment. The gold and silver nanoparticles were obtained from the nanotechnology Laboratory, Diponegoro University.

\section{Antibacterial activity of gold and silver nanoparticles against UPEC}

The antibacterial activity test was performed on a 96well microtiter plate with a total culture volume of 150 $\mu \mathrm{L}$ containing $50 \mu \mathrm{L}$ of UPEC inoculum, $50 \mu \mathrm{L}$ of PBS (Phosphate Buffer Saline), and $50 \mu \mathrm{L}$ of nanoparticle solution (gold and silver) with different concentrations $(0$, $10,20,30,40,50,60,7080,90$ and 100 ppm). Then, incubated at $37^{\circ} \mathrm{C}$ for $24 \mathrm{~h}$ under aerobic conditions. After incubation, the absorbance were measured at $\mathrm{OD}_{490}$ nm using a Microplate absorbance reader iMark (BioRad). Amoxicillin (50 ppm) was used as positive control. All assays were performed in triplicate for each treatments

\section{Antibiofilm activity of gold and silver nanoparticles against UPEC}

The antibiofilm activity test was performed on a 96well microtiter plate with a total culture volume of 150 $\mu \mathrm{L}$ containing $50 \mu \mathrm{L}$ of UPEC inoculum, $50 \mu \mathrm{L}$ of PBS, and $50 \mu \mathrm{L}$ of nanoparticle solution (gold and silver) with different concentrations $(0,10,20,30,40,50,60,7080$, 90 and $100 \mathrm{ppm})$. Then, the samples were incubated at $37^{\circ} \mathrm{C}$ for $24 \mathrm{~h}$ under aerobic conditions. After incubation, the 96-well microtiter plate was washed three times with PBS (pH 7.4) until the planktonic cells were removed and then air-dried. Next, $1 \mathrm{~mL}$ of $0.4 \%(\mathrm{w} / \mathrm{v})$ crystal solution was added and leaved for $2 \mathrm{~min}$. Then, the excess dye was removed using tissue paper. The 96-well microtiter plate was washed with sterile distilled water and then allowed to dry. Then, $1 \mathrm{~mL}$ of absolute ethanol was added to each well before reading the absorbance using a Microplate absorbance reader iMark (Bio-Rad) at $\mathrm{OD}_{490} \mathrm{~nm}$. Amoxicillin (50 ppm) was used as positive control. All assays were performed in triplicate for each treatments.

\section{Results}

This research was conducted in the Laboratory of Gastroenteritis and Salmonellosis, Institute of Tropical Disease (ITD), Airlangga University, to determine the antibacterial and antibiofilm activity of gold and silver nanoparticles on UPEC. The antibacterial test of silver (A) and gold (B) nanoparticles and the antibiofilm activity of silver (C) and gold (D) nanoparticles against UPEC was shown in Figure 1.
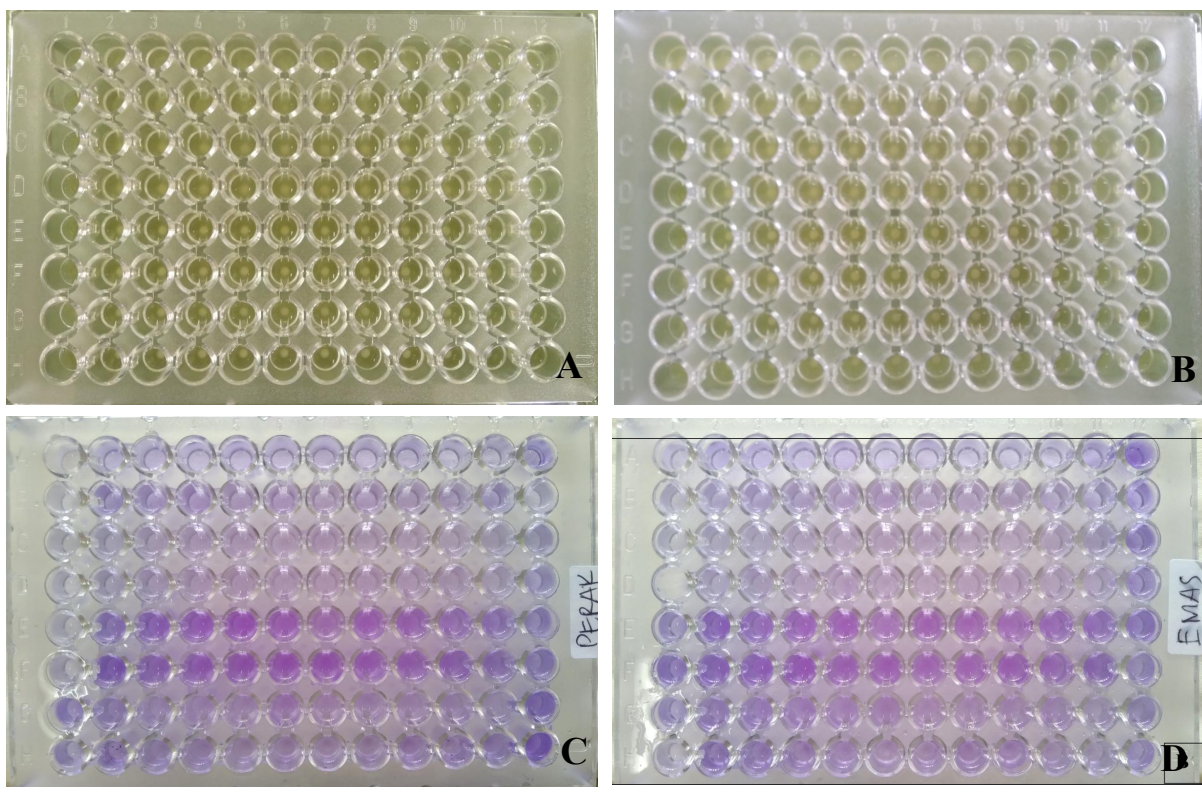

Figure 1. The antibacterial activity test of silver (A) and gold (B) nanoparticles and the antibiofilm activity of silver (C) and gold (D) nanoparticles against UPEC after $24 \mathrm{~h}$ incubation at $37^{\circ} \mathrm{C}$ 


\section{Antibacterial activity of silver nanoparticles against UPEC}

The test bacteria used in this study were isolated from urine samples in previous studies. The antibacterial activity of silver nanoparticles at various concentrations $(10,20,30,40,50,60,70,80,90$, and $100 \mathrm{ppm})$ against UPEC was evaluated by the microdilution method using Microplate absorbance reader iMark (Bio-Rad). As shown in Figure 2A, the addition of silver nanoparticles at various concentrations both affected and inhibited the growth of UPEC, as indicated by a decrease in the OD value. Of several UPEC isolates, the results showed that isolate CRE 119 had the lowest growth, followed by isolates CRE 120, CRE 94, and CRE 90. In addition, Figure 2B showed that the addition of gold nanoparticles at various concentrations also inhibited the growth of UPEC, as indicated by a decrease in OD value. Of several UPEC isolates, the results showed that isolate CRE 120 had the lowest growth, followed by isolates CRE 119, CRE 94, and CRE 90.

\section{Antibiofilm activity test of gold and silver} nanoparticles against UPEC

The antibiofilm activity test of gold and silver nanoparticles at various concentrations $(10,20,30,40$, $50,60,70,80,90$, and $100 \mathrm{ppm}$ ) against UPEC was evaluated using the microtiter plate staining method with crystal violet with measurements using Microplate absorbance reader iMark (Bio-Rad). The biofilm percentage of UPEC culture with the addition of silver and gold nanoparticles with various concentrations were shown in Figure 3.

Based on Figure 3A, the addition of silver nanoparticles at various concentrations both inhibited the formation of biofilms by UPEC. In this results, the addition of silver nanoparticles at various concentrations affected and inhibited the growth of UPEC as indicated by a decrease in the OD value. From several UPEC isolates, the results showed that CRE 118 isolate had the lowest biofilm formation ability and was followed by CRE 116, CRE 86, and CRE 73 isolates.
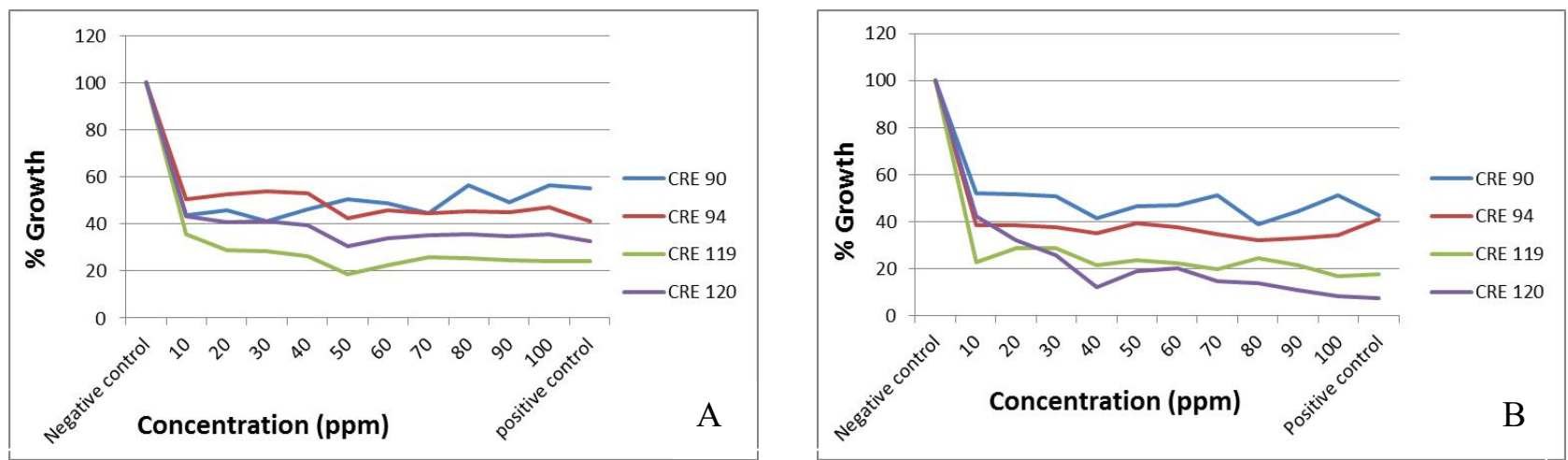

Figure 2. The growth percentage of UPEC by the addition of (A) silver and (B) gold under different concentration.


Figure 3. The biofilm percentage of UPEC by the addition of (A) silver and (B) gold under different concentration.

\section{Discussion}

Nanoparticles derived from metallic materials such as silver and metal oxides can be exploited as antibacterial agents and used in medicine. Mammalian cells can phagocytose nanoparticles and further degrade these particles by lysosomal fusion, reducing their toxicity and the resulting free radical damage. Therefore, metal nanoparticles have selectivity properties such that they can inhibit the function of bacteria that cause infection, but are also not harmful to tissue function - nanoparticles have multiple mechanisms of general toxicity to bacteria, but are not toxic to mammalian cells. Nanoparticles can bind to the bacterial cell wall and destroy the cell membrane through direct interactions or free radical production (Webster \& Taylor, 2011).

Nanotechnology has recently become a rapidly growing field with numerous biomedical applications. At the same time, silver has emerged as an antimicrobial material and disinfectant that is relatively free of adverse effects (Ortiz-Benítez et al., 2019). Nano-sized metal particles have now emerged as promising alternatives for antibiotic therapy, as they have tremendous potential to 
solve problems related to the development of multidrug resistance in pathogenic microorganisms, and therefore may also be considered as next-generation antibiotics (Dakal et al., 2016).

The antimicrobial activity of AgNPs has been studied by many researchers against various microbes, including MDR and non-MDR bacterial, fungal, and viral strains. (Dakal et al., 2016). However, several basic models for the antimicrobial activity of AgNPs have been identified. The use of sophisticated techniques such as highresolution microscopy (AFM, FE-SEM, TEM, and XRD), spectroscopy (DLS, ESR spectroscopy, fluorescence spectroscopy, inductively coupled plasma optical emission spectroscopy (ICP-MS)), molecular, and biochemical techniques have provided deep mechanistic insights into the antimicrobial action model of AgNPs (Dakal et al., 2016).

The antimicrobial effects of AgNPs involve four well-defined mechanisms: (1) adhesion of AgNPs to the cell wall and membrane surfaces, (2) penetration of AgNPs into cells and damage to intracellular structures (mitochondrion, vacuoles, ribosomes) and biomolecules (proteins, lipids, and proteins on DNA), (3) AgNPs induce cellular toxicity and oxidative stress through the generation of reactive oxygen species (ROS) and free radicals, and (4) modulation of signal transduction pathways. In addition to these four known mechanisms, AgNPs also modulate the immune system of human cells by regulating the inflammatory response, which in turn contributes to the inhibition of microorganisms (Dakal et al., 2016).

Silver nanoparticles have a broad spectrum of antibacterial, antifungal and antiviral properties. Silver nanoparticles have the ability to penetrate the cell walls of bacteria, change the structure of cell membranes and even cause cell death. Their effectiveness is due not only to their nanoscale size, but also to their large surface-tovolume ratio. They can increase the permeability of cell membranes, generate reactive oxygen species, and disrupt the replication of deoxyribonucleic acid by releasing silver ions. Although not all regulatory agencies have recognized the safety of silver nanoparticles, no systemic toxicity has been reported from ingested silver nanoparticles. A widespread concern is their potential hazard if released into the environment. However, the interaction of nanoparticles with toxic materials and organic compounds can either increase or decrease their toxicity (Ortiz-Benítez et al., 2019).

The molecular mechanism of action of bactericidal gold nanoparticles (NPs), which exhibit potent antibacterial activity against multidrug-resistant Gramnegative bacteria, was investigated using transcriptomic and proteomic approaches. Gold nanoparticles exert antibacterial activity in two main ways: first, they close the membrane potential and inhibit ATPase activity to lower ATP levels; second, they block ribosomal subunit binding to tRNA. Gold-NP enhance chemotaxis in the early phase of the reactions. The action of gold NP excludes mechanisms related to reactive oxygen species (ROS), the cause of cell death induced by most bactericidal antibiotics and other nanomaterials (Cui et al., 2012). The use of gold nanoparticles has emerged as an option to target bacterial infections; however, the mechanism responsible for bacterial cell lysis remains unclear. Specifically, it has been observed that gold nanoparticles can traverse the various structures of Streptococcus pneumoniae cells and reach the cytosol, where inclusion bodies of gold nanoparticles are important. The results of this study demonstrate a novel method for separating such inclusion bodies, allowing the analysis of biomolecules such as carbohydrates, lipids, and proteins in conjunction with gold nanoparticles. Subsequently, it was possible to separate and identify proteins associated with gold nanoparticles, which have been suggested as potential candidates for facilitating the interaction and entry of gold nanoparticles into Streptococcus pneumoniae cells. (Ortiz-Benítez et al., 2019)

The mechanism of interaction between AuNPs and bacteria was investigated using transmission electron microscopy (TEM) image analysis. The confocal laser scanning microscopy technique was used to study the cytotoxic effect of AuNPs and lasers against Corynebacterium pseudotuberculosis. The results showed that the MIC of AuNPs and AuNPs-laser combination therapy were $200 \mathrm{~g} / \mathrm{ml}$ and $100 \mathrm{~g} / \mathrm{ml}$, respectively. TEM image analysis showed that gold nanoparticles penetrate the thick walls of $C$. pseudotuberculosis and accumulate as intracellular agglomerates. Laser light increases the antimicrobial activity of gold nanoparticles at least twofold due to the combined effect of photothermia and can be used as an effective antibacterial approach against C. pseudotuberculosis (Mohamed et al., 2017).

Silver nanoparticles can bind to DNA and enzymes that control cellular respiration and other important functions, or bind to functional chemicals or receptors on cell membranes. Silver nanoparticles are highly effective against many hard-to-defeat pathogenic organisms (such as MRSA and other microorganisms). The nanoparticles exhibited a strong minimum inhibitory concentration (MIC) of 12.5 and $6.25 \mathrm{~g} / \mu \mathrm{L}$ and a minimum bactericidal concentration (MBC) of 12.5 and $12.5 \mathrm{~g} / \mathrm{mL}$ against $E$. coli and Pseudomonas aeruginosa, respectively. This means that its potent antibacterial activity demonstrated against $E$. coli and $P$. aeruginosa biofilms is expected to last for a long period of time (Singh et al., 2021).

In the form of nanoparticles, gold and silver have several advantages. In summary, the high surface area to volume ratio, ability to modify the surface, small size (less than $10 \mathrm{~nm}$ ), inertness, biocompatibility, and biosafety with relatively easy release from tissues make these nanoparticles a suitable choice for antimicrobial therapy and drug delivery. Moreover, current advances in nanotechnology, chemistry, and biotechnology enable the synthesis of AuNPs and AgNPs through simple, cheaper, and environmentally friendly methods, as well as their fine-tuning and surface modification for more effective and synergistic action. The role of AuNPs and AgNPs as antimicrobial agents involves three important steps when the nanoparticles are near the biofilm or incubated together with the biofilm (Joshi et al., 2020).

Initially, these nanoparticles interact with the surface of the biofilm after reaching it from the bulk phase. The 
activity of nanoparticles is influenced by their surface chemical structure, charge, and hydrophobicity, as well as by their interaction with lipids, LPS, or proteins from bacterial cell membranes. These factors influence the ability of nanoparticles to penetrate the biofilm. Nanoparticle penetration depends on many factors, such as biofilm maturity, chemical composition and structure of the biofilm surface, nanoparticle size, surface charge, surface chemistry, and nanoparticle concentration. After penetration, the nanoparticles migrate into the interior of the biofilm as a whole or in the form of ions (gold ions $\left(\mathrm{Au}^{+}\right)$and silver ions $\left(\mathrm{Ag}^{+}\right)$released from the nanoparticles) and interact with biofilm components and bacterial cell components (Joshi et al., 2020). Therefore, the antimicrobial activity of AuNPs and AgNPs depends on the perturbations that occur in different biofilms and bacterial components. One can modify the nanoparticles to achieve specific targets against single or multiple bacterial species. The interactions involved in biofilm surface approach, penetration, and internal migration are discussed in later chapters of this article. In general, electrostatic, hydrophobic, hydrogen bonding, and van der Waals attraction interactions are involved in all penetration processes in biofilms (Joshi et al., 2020).

The mechanism of silver nanoparticles in inhibiting bacterial biofilm formation showed that biofilm formation occurred at a bacterial concentration of $10^{6}$ $\mathrm{CFU} / \mathrm{ml}$ for susceptible $\mathrm{P}$. aeruginosa strains, while biofilm was observed at a bacterial concentration of about $10^{3} \mathrm{CFU} / \mathrm{ml}$ for resistant strains. The biofilm was then tested against different concentrations of silver nanoparticles to determine the inhibitory effect of silver nanoparticles. For the sensitive strains, $20 \mathrm{~g} / \mathrm{ml}$ silver nanoparticles inhibited growth optimally at a bacterial concentration of $10^{4} \mathrm{CFU} / \mathrm{ml}$ with an inhibition rate of 67\% (Palanisamy et al., 2014).

Similarly, silver nanoparticles inhibited biofilm formation in resistant strains at an optimal bacterial concentration of $10^{5} \mathrm{CFU} / \mathrm{ml}$ with an inhibition rate of $56 \%$. Thus, silver nanoparticles can be used as a potential alternative therapy to reduce disease severity due to $P$. aeruginosa infection (Palanisamy et al., 2014). Observations showed that biofilm formation occurred at a bacterial concentration of $10^{6} \mathrm{CFU} / \mathrm{ml}$ for susceptible $P$. aeruginosa strains, while a biofilm was evident at a bacterial concentration of approximately $10^{3} \mathrm{CFU} / \mathrm{ml}$ for resistant strains. The biofilm was then tested against different concentrations of silver nanoparticles to determine the inhibitory effect of silver nanoparticles. For the sensitive strains, $20 \mathrm{~g} / \mathrm{ml}$ silver nanoparticles inhibited growth optimally at a bacterial concentration of $10^{4} \mathrm{CFU} / \mathrm{ml}$ with an inhibition rate of $67 \%$. Similarly, silver nanoparticles inhibited biofilm formation in resistant strains at an optimal bacterial concentration of $10^{5} \mathrm{CFU} / \mathrm{ml}$ with an inhibition rate of $56 \%$. Thus, silver nanoparticles can be used as a potential alternative therapy to reduce the severity of $P$. aeruginosa infection (Palanisamy et al., 2014).

AgNP exhibited MICs of 62.5 and $125 \mathrm{~g} / \mathrm{ml}$, while their MBCs were 250 and $500 \mathrm{~g} / \mathrm{ml}$, respectively. The production of extracellular polymeric substances decreased after AgNP treatment, while cellular protein loss increased due to more severe cell membrane damage by AgNP. The percentage of biofilm inhibition was evaluated as $64 \%$ for Klebsiella pneumoniae strain MF953600 and $86 \%$ for MF953599 at an AgNP concentration of $100 \mathrm{~g} / \mathrm{ml}$. AgNPs were found to be minimally cytotoxic and safe at concentrations of 15-120 $\mathrm{g} / \mathrm{ml}$. The data evaluated in this study demonstrate that AgNPs are safe antibacterial and biofilm inhibitory compounds against MDR K. pneumoniae (Siddique et al., 2020).

Silver nanoparticles also can penetrate bacterial cell walls and subsequently change the structure of cell membranes, due to their nanoscale size. Denaturation of cytoplasmic membranes can damage organelles, and even result in cell lysis. In addition, silver nanoparticles can also be involved in signal transduction processes in bacteria. Signal transduction in bacteria is affected by the phosphorylation process that occurs on protein substrates, and nanoparticles can dephosphorylate tyrosine residues on peptide substrates. Disruption of signal transduction can lead to cell apoptosis and cell multiplication termination. The solubility of silver nanoparticles in the carrier media greatly affects its antibacterial effect and mechanism. The dissolving efficiency depends on the synthetic and processing factors, such as the intrinsic characteristics of the silver nanoparticles and the carrier medium (Ortiz-Benítez et al., 2019). In conclusions, this study showed that silver and gold nanoparticles possess antibacterial and antibiofilm effects in UPEC.

\section{Acknowledgement}

This work was supported by funding from Lembaga Penelitian dan Pengabdian Kepada Masyarakat, Wijaya Kusuma University, Surabaya, Tahun 2021.

\section{References}

Anees Ahmad, S., Sachi Das, S., Khatoon, A., Tahir Ansari, M., Afzal, Mohd., Saquib Hasnain, M., \& Kumar Nayak, A. (2020). Bactericidal activity of silver nanoparticles: A mechanistic review. Materials Science for Energy Technologies, 3, 756769. https://doi.org/10.1016/j.mset.2020.09.002

Cui, Y., Zhao, Y., Tian, Y., Zhang, W., Lü, X., \& Jiang, X. (2012). The molecular mechanism of action of bactericidal gold nanoparticles on Escherichia coli. Biomaterials, 33(7), 2327-2333. https://doi.org/10.1016/j.biomaterials.2011.11.057

Dakal, T. C., Kumar, A., Majumdar, R. S., \& Yadav, V. (2016). Mechanistic Basis of Antimicrobial Actions of Silver Nanoparticles. Frontiers in Microbiology, 7. https://doi.org/10.3389/fmicb.2016.01831

Hashimoto, M., Yanagiuchi, H., Kitagawa, H., Yamaguchi, S., Honda, Y., \& Imazato, S. (2017). Effect of Metal Nanoparticles on Biofilm formation of Streptococcus mutans. 8.

Joshi, A. S., Singh, P., \& Mijakovic, I. (2020). Interactions of Gold and Silver Nanoparticles with Bacterial Biofilms: Molecular Interactions behind Inhibition and Resistance. International Journal of Molecular Sciences, 21(20), 7658. https://doi.org/10.3390/ijms21207658

Kamat, U., Fereirra, A., Amonkar, D., Motghare, D., \& Kulkarni, M. S. (2009). Epidemiology of hospital acquired urinary tract infections in a medical college hospital in Goa. Indian Journal of Urology: IJU : Journal of the Urological Society of India, 25, 76-80. https://doi.org/10.4103/0970-1591.4554 
Medina, M., \& Castillo-Pino, E. (2019). An introduction to the epidemiology and burden of urinary tract infections. Therapeutic Advances in Urology, 11, 175628721983217. https://doi.org/10.1177/1756287219832172

Mohamed, M. M., Fouad, S. A., Elshoky, H. A., Mohammed, G. M., \& Salaheldin, T. A. (2017). Antibacterial effect of gold nanoparticles against Corynebacterium pseudotuberculosis. International Journal of Veterinary Science and Medicine, 5(1), 23-29. https://doi.org/10.1016/j.ijvsm.2017.02.003

Oliveira, F. A., Paludo, K. S., Arend, L. N. V. S., Farah, S. M. S. S., Pedrosa, F. O., Souza, E. M., Surek, M., Picheth, G., \& Fadel-Picheth, C. M. T. (2011). Virulence characteristics and antimicrobial susceptibility of uropathogenic Escherichia coli strains. Genetics and Molecular Research, 10(4), 4114-4125. https://doi.org/10.4238/2011.October.31.5

Ortiz-Benítez, E. A., Velázquez-Guadarrama, N., Durán Figueroa, N. V., Quezada, H., \& Olivares-Trejo, J. de J. (2019). Antibacterial mechanism of gold nanoparticles on Streptococcus pneumoniae. Metallomics, 11(7), 1265-1276. https://doi.org/10.1039/C9MT00084D

Palanisamy, N., Ferina, N., Amirulhusni, A., Mohd-Zain, Z., Hussaini, J., Ping, L., \& Durairaj, R. (2014). Antibiofilm properties of chemically synthesized silver nanoparticles found against Pseudomonas aeruginosa. Journal of Nanobiotechnology, 12(1), 2. https://doi.org/10.1186/1477-3155-12-2

Siddique, M. H., Aslam, B., Imran, M., Ashraf, A., Nadeem, H., Hayat, S., Khurshid, M., Afzal, M., Malik, I. R., Shahzad, M. Qureshi, U., Khan, Z. U. H., \& Muzammil, S. (2020). Effect of Silver Nanoparticles on Biofilm Formation and EPS Production of Multidrug-Resistant Klebsiella pneumoniae. BioMed Research International, 2020, 1-9. https://doi.org/10.1155/2020/6398165

Singh, P., Pandit, S., Jers, C., Joshi, A. S., Garnæs, J., \& Mijakovic, I. (2021). Silver nanoparticles produced from Cedecea sp. Exhibit antibiofilm activity and remarkable stability.

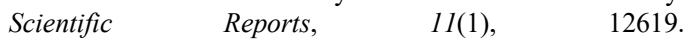
https://doi.org/10.1038/s41598-021-92006-4

Vallet-Regí, M., González, B., \& Izquierdo-Barba, I. (2019). Nanomaterials as Promising Alternative in the Infection Treatment. International Journal of Molecular Sciences, 20(15), 3806. https://doi.org/10.3390/ijms20153806

Webster, T. J. \& Taylor. (2011). Reducing infections through nanotechnology and nanoparticles. International Journal of Nanomedicine, 1463. https://doi.org/10.2147/IJN.S22021

Zhang, T. (2017). Modeling Biofilms: From Genes to Communities. Processes, 5(4), 5. https://doi.org/10.3390/pr5010005 